\title{
Tratado de Libre Comercio de America Central y Repúbica Dominicana (TLCAC-RD): ¿Qué Hay en Ello para la Agricultura de Florida? ${ }^{1}$
}

Edward A. Evans ${ }^{2}$

\section{Introducción}

El 5 de agosto del 2004, los Estados Unidos firmó el Tratado de Libre Comercio de América Central y República Dominicana (TLCAC-RD) con Costa Rica, República Dominicana, El Salvador, Guatemala, Honduras, y Nicaragua. El acuerdo, si es que es aprobado por los gobiernos de todos los países signatarios, resultará en la creación del segundo mercado más grande de exportación para los Estados Unidos en lationoamérica, después de Méjico. Será el decimocuarto mercado de exportación más grande para los Estados Unidos en el mundo, con un valor comercial (exportaciones mas importaciones) en el 2004, de más de 33 mil millones de dólares americanos. Argumentos en favor y en contra de este acuerdo se pueden encontrar virtualmente en todas partes. Aquí en Florida la reacción es mixta. El propósito de este artículo es el de resumir los elementos principales de este acuerdo, para ver que hay en ello para los productores de Florida.

\section{Elementos Principales de Tratado}

El objetivo general del acuerdo es la retirada eventual de todas las barreras al comercio (arancelarias y no arancelarias) de todos los productos con excepción de algunos considerados como sensitivos. El tratado tiene como modelo al Tratado de Libre Comercio de America del Norte (TLCAN) en el sentido de que no sólo considera cuestiones específicas de comercio tales como la reducción de aranceles, si no que también considera otras áreas tales como el de la propiedad intelectual, inversiones y servicios. Como el TLCAN, el acuerdo comprende una serie de pequeños acuerdos entre los Estados Unidos y cada uno de los socios (es decir, cada uno de los seis países del TLCAC-RD negoció separadamente un programa para dar acceso a los productos americanos). A cambio, los Estados Unidos se compromete a proveer el mismo tratamiento arancelario a todos los países socios mientras que mantiene una cuota arancelaria (CA) específica para cada país.

1. Este documento, FE574, es uno de una serie de publicaciones del Departamento de Food and Resource Economics, Servicio de Extensión Cooperativa de la Florida, Instituto de Alimentos y Ciencias Agrícolas, Universidad de la Florida. (UF/IUFAS). Este traducción del FE564. La traducción del inglés al español estuvo a cargo de Carlos Jauregui. Fecha de primera publicación: Noviembre 2005. Visite nuestro sitio web EDIS en 〈http://edis.ifas.ufl.edu>.

2. Edward A. Evans, Profesor Asistente, Departamento de Food and Resource Economics, TREC, Servicio de Extensión Cooperativa de la Florida, Instituto de Alimentos y Ciencias Agrícolas, Universidad de la Florida (UF/IUFAS), Gainesville, FL. 
En el caso de agricultura, los elementos claves del tratado caen bajo cuatro categorías: acceso al mercado, salvaguardias, medidas sanitarias y fitosanitarias, y subsidios a las exportaciones (FAS Online, http://www.fas.usda.gov).

\section{Acceso al Mercado}

Acceso al mercado es la facilidad con la que un país puede vender sus productos y servicios en otro país. Bajo este subtítulo, la liberalización (apertura a un mercado) puede ocurrir a través de una combinación en la reducción de tarifas y en la expansion de cuotas arancelarias (una tarifa con dos etapas que impone una tasa baja para cierta cuota después de la cual la tasa se incrementa). En general, las tarifas para todos los productos serán eliminadas (a excepción del azúcar en los Estados Unidos, papas y cebollas frescas que entren a Costa Rica, maíz blanco para todos los países del CAFTA con excepción de Costa Rica) dentro un periodo de eliminación gradual, con la eliminación de tarifas tomando efecto inmediatamente para algunos productos y para otros tomando efecto en un periodo de $5,10,12,15,17$, o 20 años. Para la mayoría de los productos, las reducciones ocurrirán en cuotas iguales sobre el periodo de eliminación gradual acordado. Para otros productos se establecerán cuotas arancelarias, y en algunos casos las cuotas se incrementarán sin pago de derechos para cantidades importadas específicas o para mayores cantidades sobre la cuota.

\section{Salvaguardias}

Salvaguardias son medidas precaucionarias que permiten a los países restringir importaciones si es que ellas causan daño con la provisión de que tales restricciones no sean discriminatorias y sean solo por un tiempo limitado. Para algunos productos, el tratado establece mecanismos de salvaguardia que serían implementados cuando sus importaciones dentro de un año lleguen a cierto nivel. Implementar los mecanismos de salvaguardia implica que el país puede incrementar la tarifa por un tiempo limitado. Las salvaguardias terminan cuando el periodo de eliminación gradual para ese producto termina. Por ejemplo, si un producto tiene un periodo de eliminación gradual de cinco años. los mecanismos de salvaguardia NO pueden ser aplicados el sexto año a no ser que los socios estén de acuerdo en extender el periodo más allá de los cinco años.

\section{Medidas Sanitarias y Fitosanitarias (MSF)}

Un país puede adoptar MSF, las cuales están designadas a proteger la salud humana así como la de plantas y animales. Los siete miembros están de acuerdo en utilizar el marco científico que provee la Organización Mundial del Comercio (documento EDIS, publicación FE492, Understanding the WTO Sanitary and Phytosanitary Agreement, http://edis.ifas.ufl.edu/FE492). Los siete miembros también están de acuerdo en formar un comité especial para resolver las disputas técnicas que se puedan presentar.

\section{Subsidios a las Exportaciones}

Se considera como subsidio a las exportaciones a cualquier forma de pago que ayude a los exportadores o fabricantes a bajar sus costos de exportación. Todos los participantes del tratado están de acuerdo en que no se usarán dichas medidas en el mercado de otro miembro a no ser que sea necesario para competir contra los subsidios a la exportación de un país que no es miembro.

\section{Datos Demográficos Seleccionados de los Países del TLCAC-RD}

El Cuadro 1 muestra datos demográficos seleccionados de los países del TLCAC-RD. En términos generales, los países del TLCAC-RD pueden subdividirse en dos grupos: (1) aquellos con un ingreso relativamente alto por persona, baja pobreza y alto porcentaje de alfabetismo; y (2) aquellos con un ingreso relativamente bajo por persona, alta pobreza y bajo porcentaje de alfabetismo. En el primer grupo están países como Costa Rica, República Dominicana, y El Salvador. Aquellos en el primero grupo son Guatemala, Honduras y Nicaragua. Los datos en el Cuadro 1 indican que Guatemala es el más populoso, con una población de 13.9 millones, seguido por la República Dominicana (8.7 millones), Honduras (6.7 millones), y El Salvador (6.5 millones). Costa Rica, con la población más pequeña (3.9 millones), tiene el ingreso más alto por persona de US\$8,300. Le siguen la República Dominicana y El Salvador 
con un ingreso por persona de US $\$ 6,300$ y US $\$ 4,600$ respectivamente. El ingreso relativamente bajo por persona en los países del segundo grupo sugiere el potencial por una mayor demanda por productos de alto valor (por ejemplo, carnes, frutas y verduras) conforme el ingreso por persona crezca. Guatemala tiene el primero nivel más alto de pobreza $(75 \%)$, seguido por Honduras (53\%) y Nicaragua (50\%). Estos tres países también tienen el nivel más bajo de alfabetismo. De interés es el hecho de que la agricultura es el mayor empleador en la mayoría de los países del TLCAC-RD, proveyendo el 50\% del empleo total. Esto implica que hay oportunidades tremendas para mejorar la productividad de la agricultura a través de tecnologías modernas.

\section{Beneficios para los Productores de Florida}

El Cuadro 2 muestra los principales ingresos por producto en Florida de acuerdo al Censo Agrícola del 2002. El Censo del 2002 también muestra que los ingresos totales de los principales 24 productos fueron de 6.33 mil millones de dólares. Los principales productos agrícolas en Florida son invernaderos/viveros (25.76\%), naranjas (18.46\%), caña de azúcar $(8.18 \%)$, tomates $(8.03 \%)$, productos de leche (5.63\%), y reses/terneros (5.27\%). Estos seís productos conforman el $71.33 \%$ de los ingresos brutos (Cuadro 2). Los primeros 13 productos en Florida conforman el $90.62 \%$ del total de ingresos.

En términos de valor, Florida es en primer productor de naranjas, caña de azúcar, pimientos, toronjas, frijoles verdes, maíz dulce, tangerinas, pepinos, y calabazas (Cuadro 2) y ocupa el segundo lugar en producción de cultivos de invernaderos/viveros, tomates, fresas, sandías y aguacates.

Asumiendo que las cinco categorías en el Cuadro 3 (principales exportaciones agrícolas de Florida) reflejan los grupos en los cuales los productores de Florida tienen ventaja comparativa, el análisis siguiente examina posibles oportunidades de exportación que resultarían a consecuencia de la firma del TLCAC-RD. El Cuadro 4 muestra el promedio del valor de las importaciones netas de cinco años (1999-2003). Un valor positivo en la columna de las importaciones netas indica que el país del TLCAC-RD es un importador neto de productos americanos. Un valor negativo indica lo opuesto.

En general, con respecto a las cinco categorías, la región del TLCAC-RD es un exportador neto a los Estados Unidos, con un superávit comercial de 764 millones de dólares (Cuadro 4). Sin embargo, mientras que Costa Rica, Guatemala, Honduras, y Nicaragua son exportadores netos a los Estados Unidos en las cinco categorías señaladas, la República Dominicana y el Salvador son importadores netos. En el periodo de cinco años, Costa Rica ha tenido el superávit comercial más grande con los Estados Unidos, de aproximadamente 452.68 millones, mientras que la República Dominicana fue el mayor importador neto de productos americanos, con un déficit de alrededor de 67.2 millones de dólares. En términos de países y categorías individuales, el Cuadro 4 muestra que Costa Rica es un importador neto de animales vivos, semillas para aceite, granos y semillas de Estados Unidos. La República Dominicana es un importador neto de animales vivos, carnes y menudencias comestibles; semillas para aceite, granos y semillas. El Salvador es un importador neto de animales vivos, carnes y frutas. Guatemala es un importador neto de animales vivos y carne. Honduras es un importador neto de animales vivos, carne, semillas para aceite, granos y semillas. Nicaragua es un importador neto de animales vivos, carne, semillas para aceite, granos y semillas.

El promedio de cinco años del vaor de las importaciones provenientes de los Estados Unidos y de todos los países del mundo, así como el porcentaje que representan las importaciones provenientes de Estados Unidos hacia los países del TLCAC-RD se muestran en el Cuadro 5. Definiendo una oportunidad de exportación (desde el punto de vista de Estados Unidos y Florida) como la situación en la cual estos países al momento compran de los Estados Unidos menos del $50 \%$ de sus requerimientos de importación de los productos mencionados, se pueden hacer las siguientes observaciones con respecto a la informacion presente en el Cuadro 5: 
1. El Salvador, Nicaragua y Honduras representan las mejores oportunidades para exportación. De los tres países, El Salvador, con el tercer Producto Doméstico Bruto (PDB) por persona más alto y una población mayor de 6 millones, importa solo $11.85 \%$ de estos productos de los Estados Unidos y por lo tanto representa una buena oportunidad de exportación.

2. La República Dominicana representa la peor oprtunidad de exportación porque más del $80 \%$ de los productos seleccionados ya provienen de los Estados Unidos.

3. El Salvador muestra buen potencial de exportación en las cinco categorías.

4. En los otros países del TLCAC-RD, el potencial para exportación existe en las siguientes categorías:

- Costa Rica: carne (30.54\%), verduras (12.79\%), y frutas $(41.91 \%)$.

- Guatemala: animales vivos (48.02\%), y verduras $(32.67 \%)$.

- Honduras: animales vivos (9.54\%), verduras $(30.03 \%)$, y frutas $(39.44 \%)$.

- Nicaragua: animales vivos $(11.18 \%)$, verduras $(16.34 \%)$, y frutas $(28.46 \%)$.

El nivel de acceso presente y propuesto para los productos seleccionados dentro de las cinco agrupaciones, si es que el tratado fuera implementado, se muestran en el Cuadro 6. La información en el Cuadro 6 apoya el punto de vista de que el bajo nivel de importaciones de estos productos americanos podría ser el resultado de limitaciones en el acceso al mercado.

\section{Comentarios Finales}

No hay duda de que implementando el TLCAC-RD el comercio entre los Estados Unidos y los países del TLCAC-RD se incrementará. Viendo la posición actual parece que hay ventajas en el acuerdo para los productores americanos debido a que las tarifas para los productos agrícolas que se importan a los Estados Unidos de esos países son bajas mientras que las tarifas que ellos imponen a los productos agrícolas americanos que importan son relativamente altas. Sin embargo, como con cualquier acuerdo, habrá ganadores y perdedores. En la ausencia de una bola de cristal, se puede decir que hay beneficios potenciales para los productores americanos y de Florida en la implementación del acuerdo. Las conclusiones a que se llegaron se basan en dos premisas. La primera premisa es la observación de que los productores de Florida mantienen un superavit en el comercio de los productos seleccionados a pesar de la relativa tarifa alta que los productos americanos exportados enfrentan. La segunda premisa es que removiendo las restricciones al comercio facilitará a los productores de Florida penetrar más en el mercado de los países del TLCAC-RD. Se debe mencionar que el patrón de los flujos de comercio cambia a través del tiempo y que los países que al presente importan un producto en particular, pueden, con la inversión requerida y la tecnología adecuada, convertirse en exportadores netos en un futuro cercano.

\section{Referencias}

Evans, Edward. 2005. Understanding the WTO Sanitary and Phytosanitary Agreement. Electronic Data Information Source (EDIS) FE492. Departmento de Food and Resource Economics, University of Florida, Gainesville, FL. http://edis.ifas.ufl.edu/FE492.

FAS Online. 2005. Foreign Agricultural Service, United States Department of Agriculture, Washington, D.C. http://www.fas.usda.gov.

Florida Agriculture Statistical Directory. 2004. Tallahasse, FL: Florida Department of Agriculture and Consumer Services.

Rosson, Parr. 2004. Central America Free Trade: What's at Stake for Texas Agriculture. Center for North American Studies (CNAS), Texas A\&M University, College Station, TX. http://cnas.tamu.edu/publications/PowerPoint/ CAFTATVA04.ppt.

United Nations. 2004. U.N. Commodity Trade Statistical Database. United Nations, New York, NY. http://unstats.un.org/unsd/comtrade. 
Cuadro 1. Datos demográficos seleccionados de los país del TLCAC-RD.

\begin{tabular}{|c|c|c|c|c|c|}
\hline País & Población & PDB $^{\star}$ por Persona & Pobreza & Alfabetismo & Población Agr \\
\hline & (millones) & (dólares) & $(\%)$ & (\%) & $(\%)$ \\
\hline Costa Rica & 3.9 & 8,300 & 21.0 & 96.0 & 20.0 \\
\hline El Salvador & 6.5 & 4,600 & 48.0 & 80.0 & 30.0 \\
\hline Guatemala & 13.9 & 3,900 & 75.0 & 71.0 & 50.0 \\
\hline Honduras & 6.7 & 2,500 & 53.0 & 76.0 & 34.0 \\
\hline Nicaragua & 5.1 & 2,200 & 50.0 & 68.0 & 42.0 \\
\hline República Dominicana & 8.7 & 6,300 & 25.0 & 84.0 & 17.0 \\
\hline Total/Promedio & 44.8 & 4,633 & 45.3 & 79.2 & 32.2 \\
\hline
\end{tabular}

Cuadro 2. Ingresos en efectivo más importantes por producto en Florida, 2002.

\begin{tabular}{|lrrrr|}
\hline \hline Productos & $\begin{array}{c}\text { Ingresos de } \\
\text { Florida }\end{array}$ & $\begin{array}{c}\text { Porción de } \\
\text { Ingresos }\end{array}$ & $\begin{array}{c}\text { Porción } \\
\text { Acummulada }\end{array}$ & $\begin{array}{c}\text { Rango } \\
\text { Nacional }\end{array}$ \\
\hline Iniles de $\$)$ & $1 \%$ (número $)$ \\
Naranjas & $1,629,993$ & 25.76 & 25.76 & 2 \\
Caña de azucar & $1,168,211$ & 18.46 & 44.22 & 1 \\
Tomates (frescos y procesados) & 517,925 & 8.18 & 52.40 & 1 \\
Productos de leche & 508,320 & 8.03 & 60.43 & 2 \\
Reses/Terneros & 356,184 & 5.63 & 66.06 & 14 \\
Pimientos & 333,413 & 5.27 & 71.33 & 26 \\
Papas & 218,960 & 3.46 & 74.79 & 1 \\
Pollos & 211,622 & 3.34 & 78.13 & 4 \\
Toronjas & 195,579 & 3.09 & 81.22 & 15 \\
Fresas & 183,680 & 2.90 & 84.12 & 1 \\
Frijoles verdes & 153,472 & 2.43 & 86.55 & 2 \\
Huevos & 148,764 & 2.35 & 88.90 & 1 \\
Maíz dulce & 109,012 & 1.72 & 90.62 & 12 \\
Tangerinas & 101,538 & 1.60 & 92.22 & 1 \\
\hline \hline
\end{tabular}


Cuadro 2. Ingresos en efectivo más importantes por producto en Florida, 2002.

\begin{tabular}{|c|c|c|c|c|}
\hline Productos & $\begin{array}{l}\text { Ingresos de } \\
\text { Florida }\end{array}$ & $\begin{array}{l}\text { Porción de } \\
\text { Ingresos }\end{array}$ & $\begin{array}{c}\text { Porción } \\
\text { Acummulada }\end{array}$ & $\begin{array}{l}\text { Rango } \\
\text { Nacional }\end{array}$ \\
\hline & (miles de $\$)$ & $(\%)$ & $(\%)$ & (número) \\
\hline Pepinos & 91,942 & 1.45 & 95.22 & 1 \\
\hline Aquacultura & 77,000 & 1.22 & 96.44 & - \\
\hline Sandías & 62,238 & 0.98 & 97.42 & 2 \\
\hline Calabazas & 40,611 & $0 . .64$ & 98.06 & 1 \\
\hline Maní & 35,011 & 0.55 & 98.61 & 7 \\
\hline Col (fresca) & 28,928 & 0.46 & 99.07 & 5 \\
\hline Algodón & 22,815 & 0.36 & 99.43 & 16 \\
\hline Tabaco & 18,244 & 0.29 & 99.72 & 8 \\
\hline Aguacates & 17,195 & 0.27 & 99.99 & 2 \\
\hline Total & $6,328,704$ & 100.00 & 100.00 & \\
\hline
\end{tabular}

Cuadro 3. Cinco categorías agrícolas de exportación más importantes de Florida.

\begin{tabular}{|lcc|}
\hline \hline Categoría & Valor & $\begin{array}{c}\text { Proporción de las } \\
\text { Exportaciones } \\
\text { Agrícolas Totales }\end{array}$ \\
\hline Frutas y prepardos & (millones de \$) & $(\%)$ \\
Verduras y preparados & 569 & 43.77 \\
Animales vivos y carne & 138 & 10.61 \\
Semillas para aceite, granos, semillas & 53 & 4.08 \\
Aves y productos & 37 & 2.85 \\
Total & 28 & 2.15 \\
\hline Fuente: FAS Online. & 825 & 63.46 \\
\hline \hline
\end{tabular}


Cuadro 4. Valor promedio de las importaciones provenientes de los Estados Unidos hacia los países del TLCAC-RD.

\begin{tabular}{|c|c|c|c|}
\hline & \multicolumn{3}{|c|}{ Promedio 1999-2003 } \\
\hline & $\begin{array}{c}\text { Importaciones de } \\
\text { Estados Unidos }\end{array}$ & $\begin{array}{l}\text { Exportaciones a } \\
\text { Estados Unidos }\end{array}$ & $\begin{array}{c}\text { Importaciones } \\
\text { Netas }\end{array}$ \\
\hline & & (dólares) & \\
\hline \multicolumn{4}{|l|}{ COSTA RICA } \\
\hline Animales vivos & $2,183,899$ & 439,202 & $1,744,697$ \\
\hline Carne y menudencias de carne comestibles & $2,731,830$ & $24,895,319$ & $(22,163,489)$ \\
\hline Verduras & $2,888,230$ & $50,810,486$ & $(47,922,247)$ \\
\hline Frutas & $9,547,281$ & $438,896,570$ & $(429,348,289)$ \\
\hline Semillas para aceite, granos, semillas & $51,892,351$ & $6,882,174$ & $45,010,177$ \\
\hline Total & $69,243,591$ & $521,923,751$ & $(452,680,160)$ \\
\hline \multicolumn{4}{|l|}{ REPUBLICA DOMINICANA } \\
\hline Animales vivos & $3,048,000$ & 224,000 & $2,824,000$ \\
\hline Carne y menudencias de carne comestibles & $11,548,000$ & 0 & $11,548,000$ \\
\hline Verduras & $12,834,000$ & $19,451,000$ & $(6,617,000)$ \\
\hline Frutas & $12,880,000$ & $23,684,000$ & $(10,804,000)$ \\
\hline Semillas para aceite, granos, semillas & $70,804,000$ & 551,000 & $70,253,000$ \\
\hline Total & $111,114,000$ & $43,910,000$ & $67,204,000$ \\
\hline \multicolumn{4}{|l|}{ EL SALVADOR } \\
\hline Animales vivos & $2,035,454$ & 442,881 & $1,592,573$ \\
\hline Carne y menudencias de carne comestibles & $2,593,236$ & 12,270 & $2,580,967$ \\
\hline Verduras & $1,783,147$ & $6,059,242$ & $(4,276,095)$ \\
\hline Frutas & $8,511,010$ & $2,606,154$ & $5,904,855$ \\
\hline Semillas para aceite, granos, semillas & $1,519,281$ & $1,845,720$ & $(326,439)$ \\
\hline Total & $16,442,127$ & $10,966,266$ & $5,475,861$ \\
\hline
\end{tabular}


Cuadro 4. Valor promedio de las importaciones provenientes de los Estados Unidos hacia los países del TLCAC-RD.

\begin{tabular}{|c|c|c|c|}
\hline & \multicolumn{3}{|c|}{ Promedio 1999-2003 } \\
\hline & $\begin{array}{c}\text { Importaciones de } \\
\text { Estados Unidos }\end{array}$ & $\begin{array}{l}\text { Exportaciones a } \\
\text { Estados Unidos }\end{array}$ & $\begin{array}{l}\text { Importaciones } \\
\text { Netas }\end{array}$ \\
\hline & & (dólares) & \\
\hline \multicolumn{4}{|l|}{ GUATEMALA } \\
\hline Animales vivos & $2,522,201$ & 45,418 & $2,476,783$ \\
\hline Carne y menudencias de carne comestibles & $20,448,344$ & 2,118 & $20,446,225$ \\
\hline Verduras & $4,115,981$ & $27,296,123$ & $(23,180,142)$ \\
\hline Frutas & $11,156,345$ & $208,722,499$ & $(197,566,154)$ \\
\hline Semillas para aceite, granos, semillas & $11,648,674$ & $14,148,955$ & $(2,500,281)$ \\
\hline Total & $49,891,545$ & $250,215,113$ & $(200,323,569)$ \\
\hline \multicolumn{4}{|l|}{ HONDURAS } \\
\hline Animales vivos & 724,508 & 126,226 & 598,282 \\
\hline Carne y menudencias de carne comestibles & $8,047,375$ & 412,507 & $7,634,868$ \\
\hline Verduras & $4,629,875$ & $31,865,546$ & $(27,235,671)$ \\
\hline Frutas & $6,751,668$ & $137,348,378$ & $(130,596,710)$ \\
\hline Semillas para aceite, granos, semillas & $2,561,919$ & 247,567 & $2,314,352$ \\
\hline Total & $22,715,344$ & $170,000,224$ & $(147,284,880)$ \\
\hline \multicolumn{4}{|l|}{ NICARAGUA } \\
\hline Animales vivos & 850,587 & 362,777 & 487,809 \\
\hline Carne y menudencias de carne comestibles & $2,043,235$ & $28,403,042$ & $(26,359,807)$ \\
\hline Verduras & $2,471,000$ & $3,349,263$ & $(878,263)$ \\
\hline Frutas & $1,138,070$ & $12,001,715$ & $(10,863,645)$ \\
\hline Semillas para aceite, granos, semillas & $2,073,586$ & 825,544 & $1,248,042$ \\
\hline Total & $8,576,477$ & $44,942,340$ & $(36,365,863)$ \\
\hline
\end{tabular}


Cuadro 5. Porcentaje de las importaciones provenientes de los Estados Unidos hacia los países del TLCAC-RD.

\begin{tabular}{|c|c|c|c|}
\hline & \multicolumn{3}{|c|}{ Promedio 1999-2003 } \\
\hline & $\begin{array}{l}\text { Importaciones de } \\
\text { Estados Unidos }\end{array}$ & $\begin{array}{l}\text { Importado del } \\
\text { Mundo }\end{array}$ & $\begin{array}{c}\text { Porción } \\
\text { Importada de } \\
\text { Estados Unidos }\end{array}$ \\
\hline & \multicolumn{2}{|c|}{ (dólares) } & (\%) \\
\hline \multicolumn{4}{|l|}{ COSTA RICA } \\
\hline Animales vivos & $2,183,899$ & $3,527,184$ & 61.92 \\
\hline Carne y menudencias de carne comestibles & $2,731,830$ & $8,944,866$ & 30.54 \\
\hline Verduras & $2,888,230$ & $22,573,864$ & 12.79 \\
\hline Frutas & $9,547,281$ & $22,782,549$ & 41.91 \\
\hline Semillas para aceite, granos, semillas & $51,892,351$ & $61,797,338$ & 83.97 \\
\hline Total & $69,243,591$ & $119,625,801$ & 57.88 \\
\hline \multicolumn{4}{|l|}{ REPUBLICA DOMINICANA } \\
\hline Animales vivos & $3,048,000$ & $3,141,000$ & 97.04 \\
\hline Carne y menudencias de carne comestibles & $11,548,000$ & $12,203,000$ & 94.63 \\
\hline Verduras & $12,834,000$ & $25,093,000$ & 51.15 \\
\hline Frutas & $12,880,000$ & $15,283,000$ & 84.28 \\
\hline Semillas para aceite, granos, semillas & $70,804,000$ & $76,349,000$ & 92.74 \\
\hline Total & $111,114,000$ & $132,069,000$ & 84.13 \\
\hline \multicolumn{4}{|l|}{ EL SALVADOR } \\
\hline Animales vivos & $2,035,454$ & $15,521,985$ & 13.11 \\
\hline Carne y menudencias de carne comestibles & $2,593,236$ & $34,483,505$ & 7.52 \\
\hline Verduras & $1,783,147$ & $41,905,635$ & 4.30 \\
\hline Frutas & $8,511,010$ & $41,434,629$ & 20.31 \\
\hline Semillas para aceite, granos, semillas & $1,519,281$ & $5,374,004$ & 28.27 \\
\hline Total & $16,442,127$ & $138,719,758$ & 11.85 \\
\hline
\end{tabular}


Cuadro 5. Porcentaje de las importaciones provenientes de los Estados Unidos hacia los países del TLCAC-RD.

\begin{tabular}{|c|c|c|c|}
\hline & \multicolumn{3}{|c|}{ Promedio 1999-2003 } \\
\hline & $\begin{array}{l}\text { Importaciones de } \\
\text { Estados Unidos }\end{array}$ & $\begin{array}{l}\text { Importado del } \\
\text { Mundo }\end{array}$ & $\begin{array}{l}\text { Porción } \\
\text { Importada de } \\
\text { Estados Unidos }\end{array}$ \\
\hline & (dólar & & $(\%)$ \\
\hline \multicolumn{4}{|l|}{ GUATEMALA } \\
\hline Animales vivos & $2,522,201$ & $5,252,678$ & 48.02 \\
\hline Carne y menudencias de carne comestibles & $20,448,344$ & $32,046,518$ & 63,81 \\
\hline Verduras & $4,115,981$ & $12,597,272$ & 32.67 \\
\hline Frutas & $11,156,345$ & $22,234,272$ & 50.18 \\
\hline Semillas para aceite, granos, semillas & $11,648,674$ & $17,920,826$ & 65.00 \\
\hline Total & $49,891,545$ & $90,051,567$ & 55.40 \\
\hline \multicolumn{4}{|l|}{ HONDURAS } \\
\hline Animales vivos & 724,508 & $7,593,285$ & 9.54 \\
\hline Carne y menudencias de carne comestibles & $8,047,375$ & $11,904,160$ & 67.60 \\
\hline Verduras & $4,629,875$ & $15,417,075$ & 30.03 \\
\hline Frutas & $6,751,668$ & $17,120,220$ & 39.44 \\
\hline Semillas para aceite, granos, semillas & $2,561,919$ & $4,651,395$ & 55.08 \\
\hline Total & $22,715,344$ & $56,686,135$ & 40.07 \\
\hline \multicolumn{4}{|l|}{ NICARAGUA } \\
\hline Animales vivos & 850,587 & $7,610,054$ & 11.18 \\
\hline Carne y menudencias de carne comestibles & $2,043,235$ & $3,075,243$ & 66.44 \\
\hline Verduras & $2,471,000$ & $15,125,759$ & 16.34 \\
\hline Frutas & $1,138,070$ & $3,998,669$ & 28.46 \\
\hline Semillas para aceite, granos, semillas & $2,073,586$ & $3,349,452$ & 61.91 \\
\hline Total & $8,576,477$ & $33,159,178$ & 25.86 \\
\hline
\end{tabular}


Cuadro 6. Acceso propuesto al mercado de algunos productos de interés para Florida bajo las condiciones del TLCAC-RD.

\begin{tabular}{|c|c|c|c|}
\hline Categoría & Producto & Current Tariffs & Proposed Market Access \\
\hline \multirow[t]{3}{*}{$\begin{array}{l}\text { Productos } \\
\text { Animales }\end{array}$} & Carne & $\begin{array}{l}\text { Se aplican tasas del } \\
15-30 \%, \text { pueden llegar } \\
\text { hasta el } 79 \%\end{array}$ & $\begin{array}{l}\text { Eliminación inmediata de tarifas, en todos los } \\
\text { países, a cortes de primera y selectos. La } \\
\text { excepción es la República Dominicana, la cual } \\
\text { estableció una cuota arancelaria (CA) que se } \\
\text { incrementará anualmente conforme se eliminen } \\
\text { las tarifas } \\
\text { Taraifas a otros productos de carne y } \\
\text { menudencias comestibles serán eliminadas } \\
\text { dentro de } 15 \text { años }\end{array}$ \\
\hline & Aves & $\begin{array}{l}\text { Se aplican tasas del } \\
164 \% \text {, pueden llegar } \\
\text { hasta el } 250 \%\end{array}$ & $\begin{array}{l}\text { Acceso inmediato a cuartos de pollo con pierna, } \\
\text { libre de tarifa, a través de cuotas arancelarias } \\
\text { que se incrementan anualmente. Las tarifas } \\
\text { serán eliminadas a dentro de } 17-20 \text { años } \\
\text { Se tiene planeado eliminar dentro de } 10 \text { años } \\
\text { las tarifas sobre otros productos de aves (por } \\
\text { ejemplo, alas, carne de pecho, así como carne } \\
\text { de aves deshuesada mecánicamente) }\end{array}$ \\
\hline & Productos de Leche & $\begin{array}{l}\text { Se aplican tasas del } \\
60 \% \text {, pueden llegar } \\
\text { hasta el } 100 \%\end{array}$ & $\begin{array}{l}\text { Acceso inemdiate, libre de tarifa, a través de } \\
\text { cuotas arancelarias que se incrementan cada } \\
\text { año. Todas las tarifas serán eliminadas dentro } \\
\text { de } 20 \text { años }\end{array}$ \\
\hline \multirow[t]{2}{*}{$\begin{array}{l}\text { Verduras y } \\
\text { Preparaciones }\end{array}$} & Verduras Frescas & $\begin{array}{l}\text { Se aplican tasas del } \\
15-47 \% \text {, pueden llegar } \\
\text { hasta el } 60 \%\end{array}$ & $\begin{array}{l}\text { Eliminación inmediata de tarifas, excepto las } \\
\text { tarifas sobre maíz dulce, lechuga, coliflor y } \\
\text { brócoli }\end{array}$ \\
\hline & $\begin{array}{l}\text { Verduras } \\
\text { Congelades }\end{array}$ & $\begin{array}{l}\text { Se aplican tasas del } \\
15-47 \% \text {, pueden llegar } \\
\text { hasta el } 60 \%\end{array}$ & $\begin{array}{l}\text { Eliminación inmediata de tarifas en Nicaragua y } \\
\text { eliminación dentro de 10-12 años en los otros } \\
\text { países }\end{array}$ \\
\hline \multirow[t]{3}{*}{$\begin{array}{l}\text { Frutas y } \\
\text { Preparaciones }\end{array}$} & Frutas Frescas & $\begin{array}{l}\text { Se aplican tasas del } \\
15 \% \text {, pueden llegar } \\
\text { hasta el } 150 \%\end{array}$ & $\begin{array}{l}\text { Eliminación inmediata de tarifas para } \\
\text { aproximadamente } 70 \% \text { de las frutas y nueces de } \\
\text { Estados Unidos. Eliminación de tarifas en un } \\
\text { periodo de 5-10 años para el resto }\end{array}$ \\
\hline & $\begin{array}{l}\text { Jugo Congelado de } \\
\text { Naranja }\end{array}$ & $\begin{array}{l}\text { Se aplican tasas del } \\
15 \% \text {, pueden llegar } \\
\text { hasta el } 150 \%\end{array}$ & $\begin{array}{l}\text { Eliminación inmediata de tarifas en todos los } \\
\text { países, con excepción a la República } \\
\text { Dominicana, la que las eliminará dentro de } 15 \\
\text { años }\end{array}$ \\
\hline & Jugo de Toronja & $\begin{array}{l}\text { Se aplican tasas del } \\
20-60 \% \text {, pueden llegar } \\
\text { hasta el } 150 \%\end{array}$ & Eliminación inmediata de tarifas \\
\hline
\end{tabular}

